\title{
Minimally Invasive Transforaminal Lumbar Interbody Fusion: Technical Note
}

\author{
Gabriel C. Tender*,1 and John K. Ratliff ${ }^{2}$ \\ ${ }^{I}$ Department of Neurosurgery, Louisiana State University Health Sciences Center, 2020 Gravier Street, Suite 326, New \\ Orleans, LA 70112, USA \\ ${ }^{2}$ Department of Neurosurgery, Thomas Jefferson University, 909 Walnut, 2nd Floor, Philadelphia, PA 19147, USA
}

\begin{abstract}
Minimally invasive techniques for lumbar interbody fusion have become increasingly popular recently. A limitation of these procedures is the restricted field of view, which requires a thorough understanding of the three-dimensional spinal landmarks and appropriate technical skills. We outline the operative steps used in said cases and provide illustrative video and figures.
\end{abstract}

Keywords: Low back pain, surgical approach, transforaminal lumbar interbody fusion.

\section{INTRODUCTION}

Lumbar fusion has been shown to provide good longterm results in selected patients with low back pain due to degenerative disease [1]. Traditional instrumented lumbar fusion procedures may be associated with extensive paraspinal muscle damage and significant blood loss [2-5]. Minimally invasive approaches offer multiple advantages: decreased exposure-related muscle injury, decreased blood loss, decreased postoperative pain, and shorter hospital stay [6-12]. Another operative advantage is the favorable angle of approach, allowing for better disc space preparation with minimal neural element retraction. Intraoperative dural tears, common during surgeons' training phase, may be less likely to lead to a cerebrospinal fluid fistula, due to the sealing effect of the paraspinal musculature. We present an operative algorithm for minimally invasive transforaminal lumbar interbody fusion exposure. We hope via illustration of our approach to minimally invasive lumbar interbody fusion to foster further development and refinement of minimally invasive operative techniques. Review of this algorithm may be useful for residents, fellows, or other physicians in training.

\section{OPERATIVE TECHNIQUE}

After general anesthesia via endotracheal intubation is achieved, the patient is placed on a Jackson or other radiolucent table in prone position, with the arms carefully positioned to prevent a brachial plexus stretch injury. The lumbar area is prepared and draped in the usual sterile fashion, and intraoperative fluoroscopy is brought into the operative field. Patient positioning allows for the $\mathrm{C}$-arm to be moved towards the head of the bed while maintaining the sterile conditions. The pars interarticularis of the target level is identified fluoroscopically; for example, the pars of L4 will lie

*Address correspondence to this author at the Department of Neurosurgery, Louisiana State University, Health Science Center, 1542 Tulane Avenue New Orleans LA 70112, USA; E-mail: GTende@1suhsc.edu between the L3-4 and L4-5 facet joints [13]. A guidewire is docked upon the targeted lumbar lamina, and then an initial tissue dilator is passed; these steps are visualized with continuous fluoroscopy. The guidewire is removed and initial tissue dissection is completed with either a small tissue dilator or cob-type percutaneous dissector. Conversely, initial dissection may be completed via a small skin and fascial incision under direct vision, followed by placement of the initial tissue dilators.

Serial dilators are then used in order to place the final tubular retractor. The tubular retractor is immobilized via an adjustable arm and maintains exposure for the remainder of the case (Fig. 1). The placement of skin incision depends on the size of the patient: with more obese patients, a farther lateral incision may be required. An "en face" fluoroscopic view of the targeted vertebral body pedicles may help provide site for skin incision.

The angle between the axis of the tubular retractor with the vertical plane should be about 15 to 30 degrees for posterior lumbar interbody fusions (PLIFs), or 25 to 40 degrees for transforaminal lumbar interbody fusions (TLIFs). A greater angle of approach may facilitate pedicle screw placement via the working channel after cage insertion.

Of particular importance is the placement of lumbar fascia incision, which is the limiting factor in the mobilization ("wanding") of the tubular retractor during the procedure. The incision in the fascia should be medial to, and longer (in the cranio-caudal direction) than the skin incision (the more obese the patient, the more medial and longer the fascial incision).

Final exposure is similar across different tubular retractor platforms and usually allows for visualization of 22 to 26 $\mathrm{mm}$ in the long axis (Fig. 2). Despite the use of serial dilators, the final tubular retractor seldom completely clears the paraspinal musculature off posterior bony elements. Bipolar or Bovie coagulation can be used to remove this small amount of remaining muscle tissue. The initial exposure of the bony elements should allow for identification of the pars 

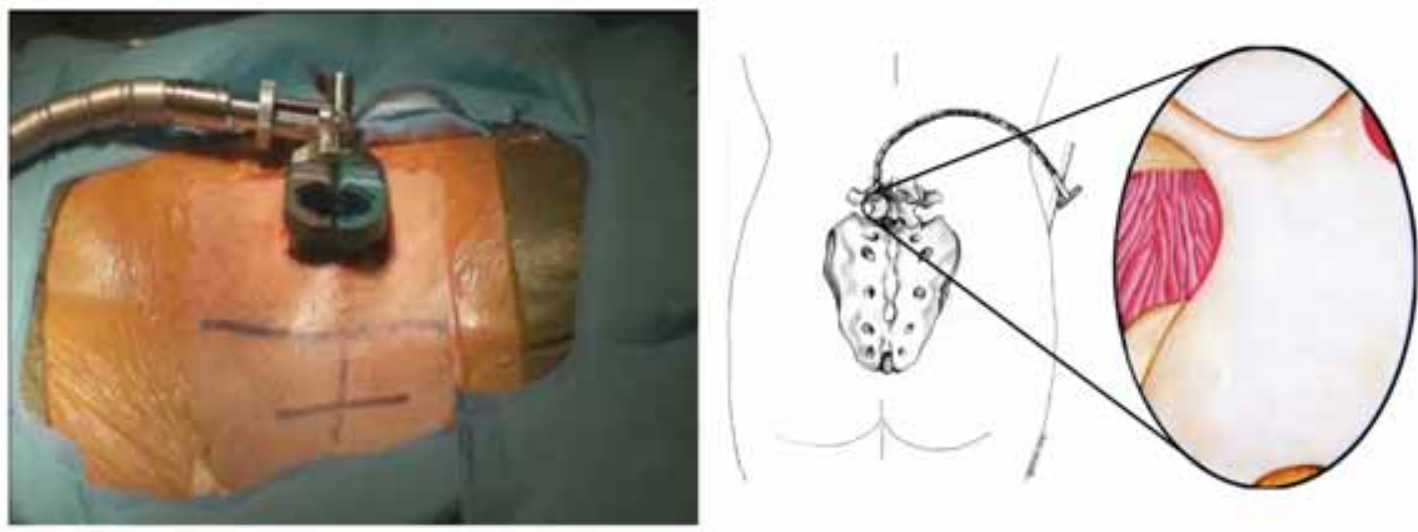

Fig. (1). Intraoperative image (left) and artist's depiction (right) of the tubular retractor immobilization via an adjustable arm for the minimally invasive exposure.
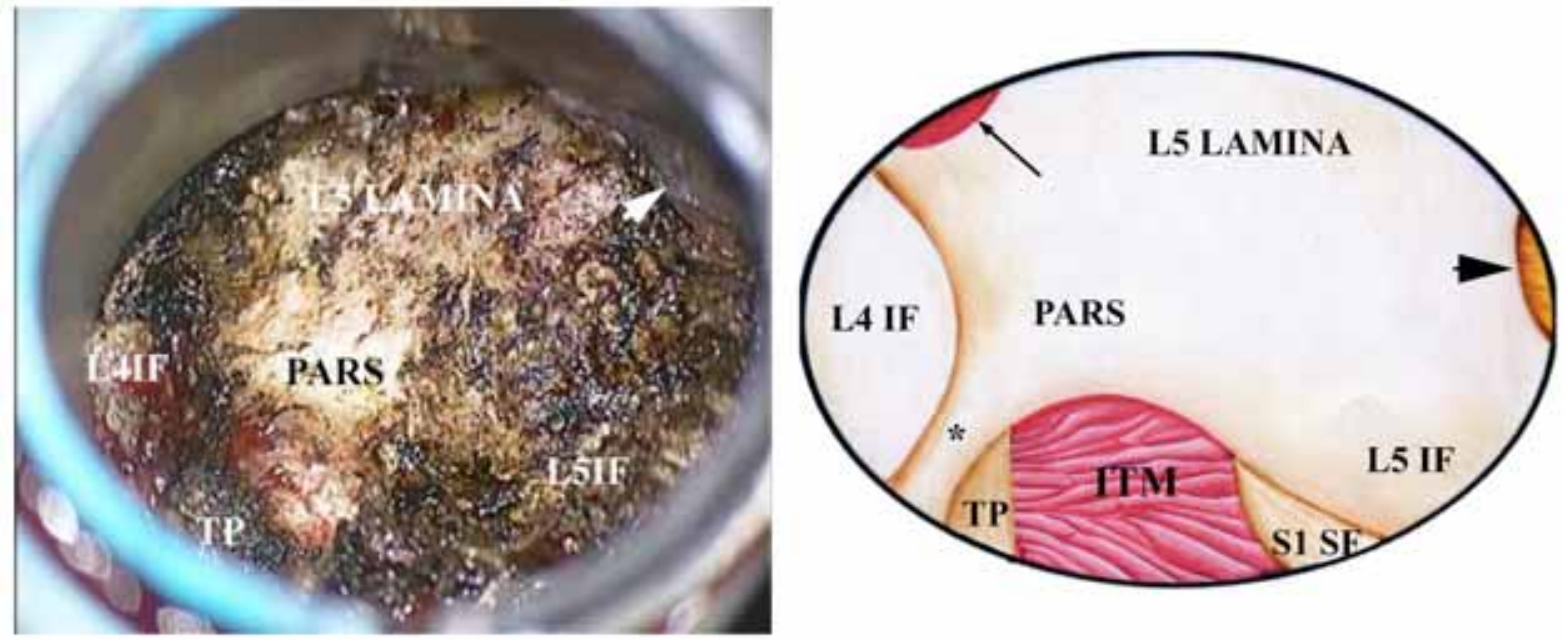

Fig. (2). Intraoperative image (left) and artist's depiction (right) of the initial exposure after removal of the muscle fiber remnants. The pars interarticularis is identified as the "valley between two hills" (L4IF = L4 inferior facet, TP = L5 transverse process, PARS = L5 pars interarticularis, ITM = intertransverse membrane and muscle, S1SF = S1 superior facet, L5IF = L5 inferior facet).

interarticularis as the "valley between two hills" - the superior and inferior facet joints. If the exposure is wide enough, the cranial and caudal edges of the lamina can also be identified. Medially, the retractor is "docked" against the lamina and spinous process junction - the vertical angle of the spinous process will limit any further medial mobilization. Lateral fluoroscopic images should be used to confirm the cranial and caudal limits of the desired exposure, corresponding to the inferior edge of the superior pedicle and the superior edge of the inferior pedicle, respectively.

A common technical error is to drill too much of the pars interarticularis cranially, perhaps violating the superior pedicle, while resecting too little caudally, and not achieving adequate disc exposure. The relatively constant posterior lumbar artery is encountered at the junction of the pars with the inferior facet, and has to be controlled by bipolar coagulation.

Bony removal may be achieved via use of the high-speed drill with a matchstick-type burr on a curved shaft. Some bone removal can be done with Kerrison rongeurs, and the harvested bone can be later used to augment the interbody and intertransverse fusion. Bone drilling should start in the neutral zone, around the junction between the pars and the inferior facet, and proceed from a lateral to medial direction. As the exposure approaches the lamina, typical cortical bone - cancellous bone - cortical bone anatomy is encountered. By starting in the neutral zone, penetration of the deep cortical layer of the lamina will expose the underlying yellow ligament (Fig. 3), thus preventing a dural tear. Another safety measure is to keep the drill at an angle of 30 to 45 degrees with the vertical axis, so that only the non-fluted tip of the matchstick drill bit comes into contact with underlying structures (i.e., if the exposure is too far cranially and the dura mater lies under the deep cortical layer of the lamina, the above described technique will reduce the risk of the fluted part of the drill bit generating an incidental durotomy).

Once the yellow ligament is encountered, bony removal is extended in the medial part of the exposure to the desired cranial and caudal limits, generally the space between the superior and inferior pedicles on lateral fluoroscopic image. This usually corresponds, in the cranial direction, to the end of the yellow ligament, so that a small part of the dural surface is exposed. In the caudal direction, bony removal often leads to the complete detachment of the inferior facet, which 

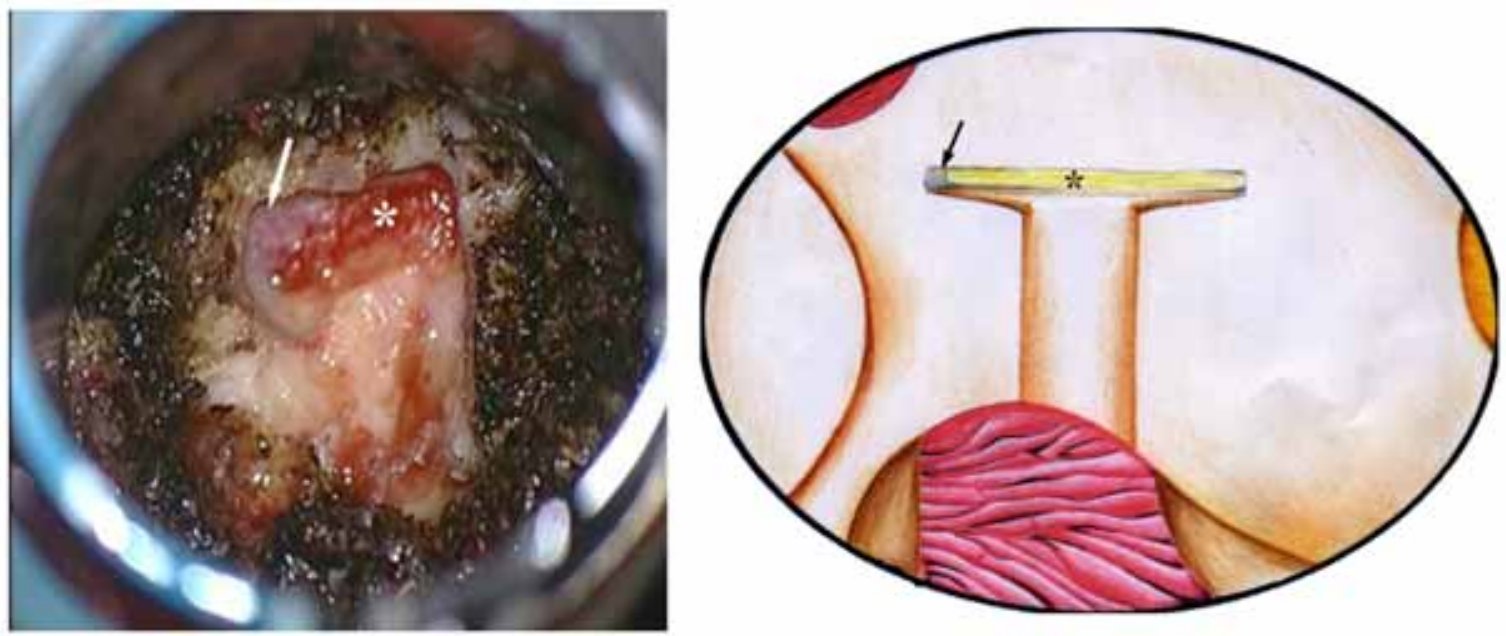

Fig. (3). Intraoperative image (left) and artist's depiction (right) of the initial bony removal with the high-speed drill. Going from lateral to medial, the yellow ligament is encountered $(*)$. Following the yellow ligament cranially, dura mater (arrow) is exposed and usually defines the cranial limit of bone removal. The caudal limit is inferred fluoroscopically from the inferior pedicle projection.

can then be removed with a pituitary rongeur, morselized, and used as local bone graft.

Further exposure is performed with the high-speed drill in a medial to lateral direction to complete the bony exposure; the deep portion of the posterior vertebral elements can be removed with a Kerrison rongeur. In the caudal portion of the exposure, once the inferior articulating process of the superior facet is removed, the smooth articulating surface of the superior articulating process of the subjacent facet is encountered. For optimal disc exposure, the cranial tip of the subjacent facet can be removed, either with the high-speed drill or the Kerrison rongeur. At the end of this step, the edge, medial and lateral borders of both the superior and inferior pedicles can be felt with a blunt nerve hook.

Next, the yellow ligament is removed. Since the cranial end of the ligamentum flavum has already been exposed, a small upbiting curette can be inserted between the ligament and the underlying dura mater, followed by a larger size Kerrison rongeur that may complete the ligament removal over the exposed surface. At this time, if medial retraction of the thecal sac is deemed necessary for cage placement, the highspeed drill or Kerrison rongeur is used to extend the medial laminotomy to the lamina - spinous process junction (i.e., the medial limit of the exposure provided by the tubular retractor). Wanding of the working channel slightly medially may provide greater exposure of the medial portion of the vertebral lamina.

After the removal of the yellow ligament, the epidural venous plexus is exposed (Fig. 4). In the cranial part of the extraforaminal space, a constant artery (the arcade of Dunsker [14]) is identified as a bridge over the spinal nerve. This vessel (or vessels) must be isolated, coagulated, and sharply transected. An excellent landmark for localizing the exiting spinal nerve is the corresponding pedicle; the nerve is generally in contact with and running along the caudal edge of the pedicle. The disc may be safely exposed along the medial, caudal aspect of the foraminal exposure, away from the exiting spinal nerve.

Once the epidural veins and the arcade of Dunsker [14] are coagulated and sharply transected, the annulus fibrosus
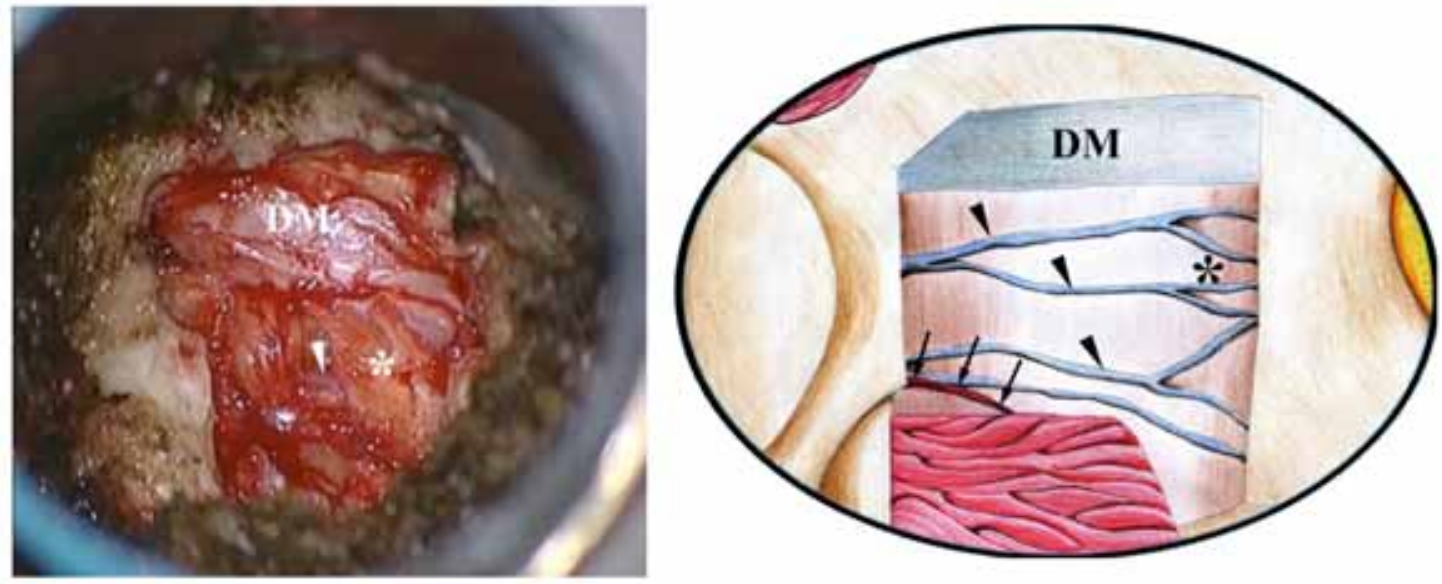

Fig. (4). Intraoperative image (left) and artist's depiction (right) of the exposure after removal of the bone and yellow ligament. Dura mater (DM) of the thecal sac is exposed medially, the arcade of Dunsker (arrows) overlie the exiting spinal nerve, and the epidural veins (arrowheads) obscure the exposure. Bipolar coagulation of these veins is safest to start in the medial caudal quadrant $(*)$. 

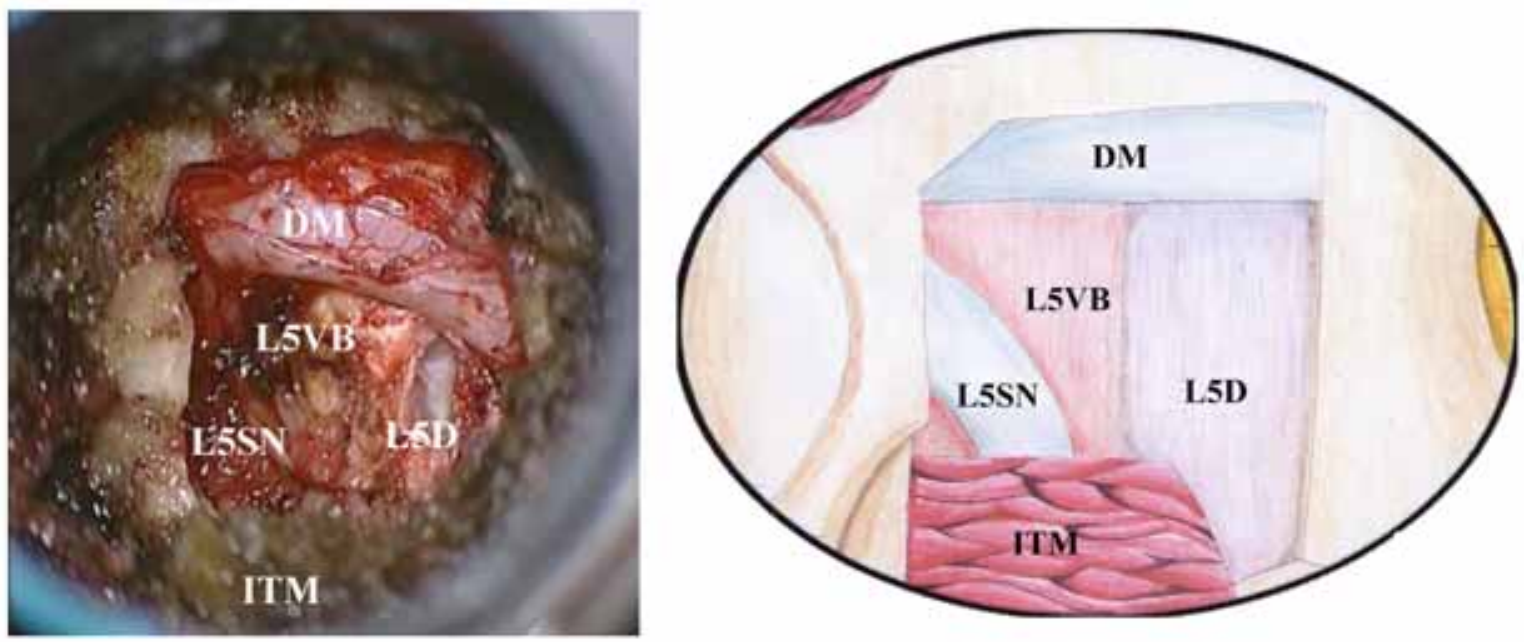

Fig. (5). Intraoperative image (left) and artist's depiction (right) of the exposure of the disc of interest and the exiting spinal nerve. The annulotomy can be extended medially by retracting the dura; if lateral exposure is needed, the lateral facet can also be removed. (DM $=$ dura mater, $\mathrm{L} 5 \mathrm{VB}=\mathrm{L} 5$ vertebral body, $\mathrm{L} 5 \mathrm{SN}=\mathrm{L} 5$ spinal nerve, L5D = L5 disc, ITM = intertransverse membrane and muscle).

and the exiting spinal nerve are exposed (Fig. 5). An annulotomy may be generated with a bayoneted, retractable scalpel blade. We place the lateral edge of the annulotomy medial enough so that the spinal nerve remains at a safe distance. When larger cages are required, the thecal sac may be gently mobilized and retracted medially, if an adequate laminotomy has been performed. A common error is to place the annulotomy too far laterally, which may lead, at the time of cage insertion, to compression of the exiting spinal nerve against the superior pedicle.

After the annulotomy is completed, disc material is removed using a variety of straight and angled curettes, disc space shavers, and paddle distractors of progressively larger sizes. The disc and endplate preparation must be thorough; fusion rate will depend upon adequate endplate decortication. An implantable cage or bone graft provides substrate for the interbody fusion.
Due to the naturally concave shape of the vertebral endplates, often compounded by large osteophytes, it is often difficult to insert an appropriately sized cage through the narrow opening at the level of the annulotomy. Several maneuvers can be used to facilitate appropriate cage sizing. Use of "bullet nose" shaped cages allows for progressive distraction of the two endplates by the cage itself, as it is inserted. Distraction of the opposite side of the vertebral elements via percutaneous pedicle instrumentation may maintain open the ipsilateral annulotomy, allowing for positioning of a larger intervertbral device. Intervertebral distraction may be garnered via rotation of blunt paddle distractors within the vertebral interspace. The distraction may be maintained via provisional tightening of contralateral percutaneous fixators. Small osteotomes can be used to remove part of the bony edges of the annulotomy; however this maneuver may presidpose to cage retropulsion. Finally, we prefer to make the final turn with the largest part of the shaver paddle at the
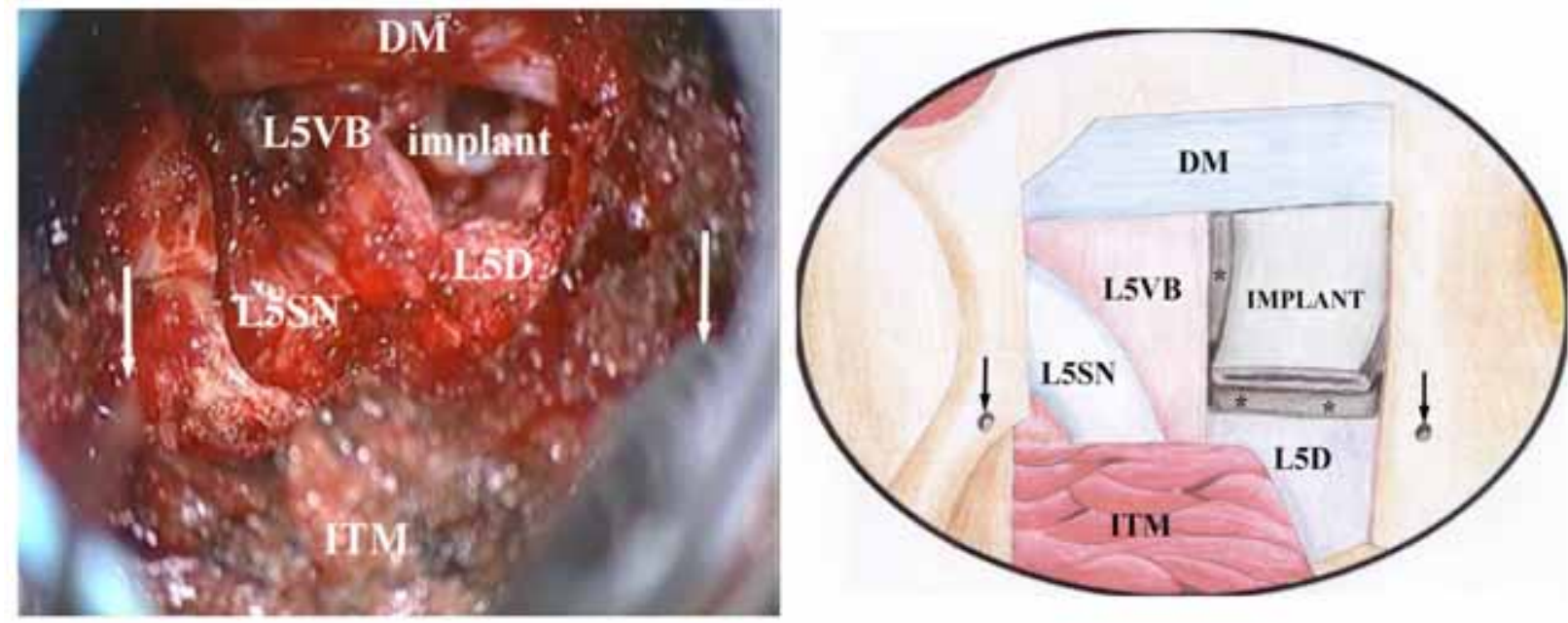

Fig. (6). Intraoperative image (left) and artist's depiction (right) of the disc exposure and implant insertion. The largest size implant should be inserted in the interspace $(*)$. Pedicle screw insertion points (arrows) can be identified by directly palpating the pedicles. (DM $=$ dura mater, L5VB = L5 vertebral body, IMPLANT = interbody implant, L5SN = L5 spinal nerve, L5D = L5 disc, ITM = intertransverse membrane and muscle). 

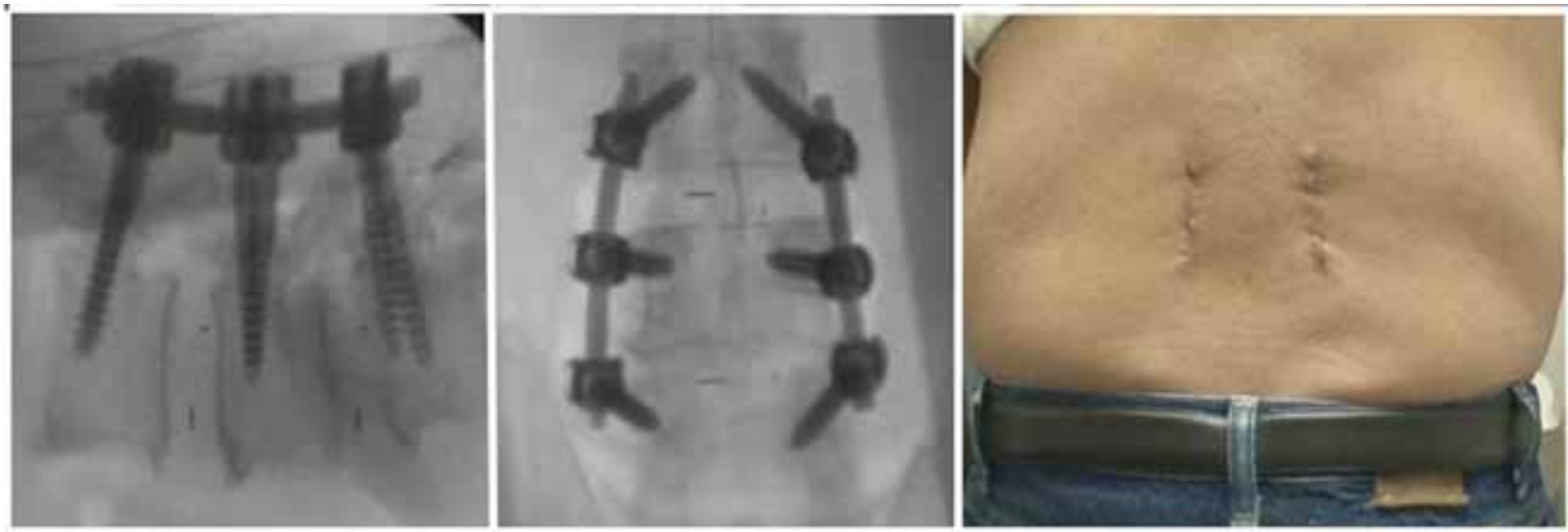

Fig. (7). Lateral (left)and anteroposterior (middle) lumbar intraoperative fluoroscopic images showing the instrumented L3-L4-L5 fusion. Right, Postoperative incisions at the 2-week follow-up visit.

superficial level of the annulotomy, while protecting the thecal sac and exiting spinal nerve, so that a cage of the same size will easily fit through the opening.

An interbody spacer provides substrate for arthrodesis. Commercial vertebral body replacement devices, allograft spacers, or autograft bone may be used. These agents may be mixed with locally harvested bone obtained during the laminotomy. The interbody spacer is inserted in the disc space. The largest technically feasible spacer should be used. The size of the spacer can be inferred from the largest diameter shaver or distractor paddle used to prepare the endplates and distract the vertebral bodies. Optimal distraction of the interbody space may be confirmed via fluoroscopic images. According to the surgeon preference, either a PLIF or TLIF cage can be used, depending on the angle of insertion. Protection of the thecal sac and the exiting spinal nerve during this step is mandatory.

Entry points for ipsilateral pedicle screws are easy to determine, since both pedicles can be palpated with a nerve hook or similar instrument (Fig. 6). The entry point for the superior pedicle should correspond to the junction between the pars, superior facet, and transverse process. We prefer to complete the preparation of both screw trajectories (including placement of the entry point using the high-speed drill, cannulation of the pedicle using the pedicle finder and tap, and palpation of the screw trajectory with the ball probe) before actually inserting the screws, since the working space is limited. Hemostasis after preparation of the first screw trajectory can be obtained through placement of a small piece of Gelfoam in the created screw path. Once both screws are inserted, the rod is placed on top of the screw heads and then tightened in position with the appropriate caps. Compression or distraction may be applied, if necessary. The tubular retractor is then removed and the wound is closed in anatomical layers. Final antero-posterior and lateral views of the construct can be obtained (Fig. 7).

\section{CONCLUSION}

We present illustrative video and pictures for the minimally invasive instrumented lumbar interbody fusion technique. A stepwise approach may limit the incidence of technique related perioperative complications and may be useful for spine surgeons in training.

\section{ACKNOWLEDGEMENTS}

We would like to thank the artist, Ms. Kirsten Berberovich, for the depiction of the operative steps for unilateral transforaminal interbody fusion (Figs. 1-6, right).

\section{SUPPLEMENTARY MATERIAL}

Supplementary material is available on the publishers Web site along with the published article.

\section{REFERENCES}

[1] Fritzell P, Hagg O, Wessberg P, Nordwall A. 2001 Volvo Award Winner in Clinical Studies: Lumbar fusion versus nonsurgical treatment for chronic low back pain: a multicenter randomized controlled trial from the Swedish Lumbar Spine Study Group. Spine 2001; 26(23): 2521-32.

[2] Kawaguchi Y, Yabuki S, Styf J, et al. Back muscle injury after posterior lumbar spine surgery. Topographic evaluation of intramuscular pressure and blood flow in the porcine back muscle during surgery. Spine 1996; 21(22): 2683-8.

[3] Kawaguchi Y, Matsui H, Tsuji H. Back muscle injury after posterior lumbar spine surgery. A histologic and enzymatic analysis. Spine 1996; 21(8): 941-4.

[4] Sihvonen T, Herno A, Paljarvi L, Airaksinen O, Partanen J, Tapaninaho A. Local denervation atrophy of paraspinal muscles in postoperative failed back syndrome. Spine 1993; 18(5): 575-81.

[5] Styf JR, Willen J. The effects of external compression by three different retractors on pressure in the erector spine muscles during and after posterior lumbar spine surgery in humans. Spine 1998; 23(3): 354-8.

[6] Fessler RG. Minimally invasive spine surgery. Neurosurgery 2002; 51(5 Suppl): Siii-Siiv.

[7] Fessler RG, O'Toole JE, Eichholz KM, Perez-Cruet MJ. The development of minimally invasive spine surgery. Neurosurg Clin N Am 2006; 17(4): 401-9.

[8] Foley KT, Holly LT, Schwender JD. Minimally invasive lumbar fusion. Spine 2003; 28(15 Suppl): S26-S35.

[9] Guiot BH, Khoo LT, Fessler RG. A minimally invasive technique for decompression of the lumbar spine. Spine 2002; 27(4): 432-8.

[10] Holly LT, Schwender JD, Rouben DP, Foley KT. Minimally invasive transforaminal lumbar interbody fusion: indications, technique, and complications. Neurosurg Focus 2006; 20(3): E6.

[11] Khoo LT, Palmer S, Laich DT, Fessler RG. Minimally invasive percutaneous posterior lumbar interbody fusion. Neurosurgery 2002; 51(5 Suppl): S166-1. 
[12] Schwender JD, Holly LT, Rouben DP, Foley KT. Minimally invasive transforaminal lumbar interbody fusion (TLIF): technical feasibility and initial results. J Spinal Disord Tech 2005; 18: S1-S6.

[13] Tender GC, Baratta RV, Voorhies RM. Unilateral removal of pars interarticularis. J Neurosurg Spine 2005; 2(3): 279-88.
[14] Viswanathan R, Swamy NK, Tobler WD, Greiner AL, Keller JT, Dunsker SB. Extraforaminal lumbar disc herniations: microsurgical anatomy and surgical approach. J Neurosurg 2002; 96 (2 Suppl): 206-11.

Received: December 11, 2008

Revised: April 09, 2009

Accepted: April 21, 2009

() Gabriel C. Tender; Licensee Bentham Open.

This is an open access article licensed under the terms of the Creative Commons Attribution Non-Commercial License (http://creativecommons.org/licenses/by-nc/3.0/) which permits unrestricted, non-commercial use, distribution and reproduction in any medium, provided the work is properly cited. 